\title{
VOLUME ESTIMATES FOR TUBES AROUND SUBMANIFOLDS USING INTEGRAL CURVATURE BOUNDS
}

\author{
YOUSEF K. CHAHINE
}

\begin{abstract}
We generalize an inequality of E. Heintze and H. Karcher [8] for the volume of tubes around minimal submanifolds to an inequality based on integral bounds for $k$-Ricci curvature. Even in the case of a pointwise bound, this generalizes the classical inequality by replacing a sectional curvature bound with a $k$-Ricci bound. This work is motivated by the estimates of PetersenShteingold-Wei for the volume of tubes around a geodesic [12] and generalizes their result. Using similar ideas we also prove a Hessian comparison theorem for $k$-Ricci curvature which generalizes the usual Hessian and Laplacian comparison for distance functions from a point and give several applications.
\end{abstract}

\section{INTRODUCTION}

The geodesic tube of radius $r$ around a closed submanifold $\Sigma^{m}$ of a Riemannian manifold $M^{n}$, denoted $T(\Sigma, r)$, is the set of all points whose distance to $\Sigma$ is at most $r$. In this paper, we give upper bounds for the volume of $T(\Sigma, r)$ based on $L^{p}$ norms of the negative part of the $k$-Ricci curvature of $M$. For $p=\infty$, we prove that the well-known estimate of E. Heintze and H. Karcher based on pointwise sectional curvature bounds requires only $k$-Ricci bounds (Theorem 4.1). The main result is the case $p<\infty$, where we give the first estimates for the volume of tubes around submanifolds of general codimension using integral curvature bounds (Theorem 1.1).

The $k$-Ricci curvature interpolates between sectional curvature and Ricci curvature by taking an average of sectional curvatures over a $k$-dimensional subspace of the tangent space. Specifically, given a unit vector $u$ tangent to $M$ and $k$ dimensional subspace $\mathcal{V}$ of the tangent space orthogonal to $u$ the $k$-Ricci curvature of $(u, \mathcal{V})$ is defined by

$$
\operatorname{Ric}_{k}(u, \mathcal{V})=\sum_{i=1}^{k}\left\langle R\left(e_{i}, u\right) u, e_{i}\right\rangle
$$

where $e_{1}, \ldots, e_{k}$ form an orthonormal basis of $\mathcal{V}$. Notice that $R i c_{n-1}$ is equivalent to the Ricci curvature and $R i c_{1}$ is equivalent to sectional curvature. We say that a manifold has $k$-Ricci curvature bounded below by $k H$ for some constant $H$ if $\operatorname{Ric}_{k}(u, \mathcal{V}) \geq k H$ for all unit vectors $u \in T M$ and $k$-dimensional subspaces $\mathcal{V} \perp u$.

The earliest global results using $k$-Ricci lower bounds as a partial positivity condition for curvature were obtained by Wu [23], Shen [19], and Shen-Wei [20], though the relationship between $k$-Ricci curvature and volume had been considered previously by Bishop and Crittenden [2, p. 253]. A signficant literature has

2010 Mathematics Subject Classification. Primary 53C20. 
since developed which bridges a gap between the global results based on sectional curvature bounds and those based on Ricci curvature bounds [21, 16, 24, 7, 9].

In a different direction, it has recently been shown that some global results still hold even without pointwise bounds, provided the part of the curvature which violates a pointwise bound is small in an $L^{p}$ sense $[4,25,13,12,15,1]$. To make this precise, for a real-valued function $f$ let $f_{+}=\max \{f, 0\}$ and $f_{-}=\max \{-f, 0\}$ denote the positive and negative parts of $f$, respectively. Given a manifold $(M, g)$, let $\rho_{k}(x)$ denote the minimum of $\operatorname{Ric}_{k}(u, \mathcal{V})$ where $u \in T_{x} M$ is a unit tangent vector at $x$ and $\mathcal{V}$ is a $k$-dimensional subspace orthogonal to $u$. For a fixed constant $H$ we may then consider the norms

$$
\left\|\left(\rho_{k}-H\right)_{-}\right\|_{p}=\left(\int_{M}\left(\rho_{k}-H\right)_{-}^{p} d v o l_{g}\right)^{1 / p}
$$

which measure the amount of $k$-Ricci curvature below $H$.

Volume estimates for geodesic balls and tubes around hypersurfaces using bounds on $\left\|\left(\rho_{n-1}-H\right)_{-}\right\|_{p}$ have been obtained by Gallot [4], Yang [25], and Petersen-Wei [13]. The latter established a relative volume comparison using integral curvature bounds and has numerous applications. Volume estimates for tubes around geodesics using integral curvature bounds were also obtained by Petersen-ShteingoldWei [12] and used to generalize Cheeger's lemma and the Grove-Petersen finiteness theorem to manifolds with integral curvature bounds.

This last work illustrates the increase in difficulty in the case that $\Sigma$ has arbitrary codimension. In particular, estimates were needed for certain quadratic invariants of the Hessian of the distance function which were completely new to comparison geometry [12, Lemma 3.1]. Our methods are based on the ideas of [12]; however, in that work a number of simplifications were employed specific to the 1-dimensional case which make modification to general codimension nontrivial. Indeed, if one naively adapts the arguments of [12] the resulting volume estimates require stronger assumptions on the curvature of $M$ and on the second fundamental form of $\Sigma$ than are necessary.

In Section 5, we show how these types of estimates generalize to tubes around minimal submanifolds of all dimensions. Specifically, we prove

Theorem 1.1. Let $M^{n}$ be a complete Riemannian manifold and let $\Sigma^{m} \subset M$ be an $m$-dimensional closed minimal submanifold with $0<m<n-1$ and put $k=\min \{m, n-m-1\}$. If $H \leq 0$ and $p>n-k$ then

$$
\operatorname{vol}(T(\Sigma, r)) \leq\left(w(r)^{n-m-1}+2^{p / \alpha}\left\|\left(\rho_{k}-H\right)_{-}\right\|_{p}^{\beta p} w(r)^{p}\right) e^{\kappa r^{2 \alpha}}
$$

where $\alpha=\frac{n-k-1}{n-k}, \beta=\frac{1}{n-m-1}-\frac{1}{p}$,

$$
\begin{aligned}
w(r) & =\left(\frac{\alpha}{n-m-1}\right)^{\frac{1}{n-k-1}}\left(\operatorname{vol}\left(\mathbb{S}^{n-m-1}\right) \operatorname{vol}(\Sigma) r^{n-m}\right)^{\frac{1}{n-m-1}}+\delta\left\|\left(\rho_{k}-H\right)_{-}\right\|_{p}^{1-\beta} r^{2}, \\
\text { and } \kappa & =(\delta|H|)^{\alpha} /(2 \alpha) \text { with }
\end{aligned}
$$

$$
\delta=4(n-k-1)+\frac{4}{k}\left(\frac{2 p-1}{p-n+k}\right) .
$$

Remark 1.2. As mentioned above, estimates when $\Sigma$ is a point or a hypersurface have already been obtained in $[4,13]$ so we do not repeat this case. 
Remark 1.3. In the case of a pointwise lower bound $R i c_{m} \geq 0$ (i.e. $\left\|\left(\rho_{m}\right)_{-}\right\|_{p}=0$ ) with $m \leq n-m-1$ the estimate above reduces to

$$
\operatorname{vol}(T(\Sigma, r)) \leq \frac{1}{n-m} \operatorname{vol}(\Sigma) \operatorname{vol}\left(\mathbb{S}^{n-m-1}\right) r^{n-m} .
$$

In particular, this shows that the Heintze-Karcher estimate [8, Corollary 3.3.1] holds for tubes around minimal submanifolds assuming only a $k$-Ricci lower bound in place of a sectional curvature lower bound. In fact, in Theorem 4.1 below we show that the Heintze-Karcher estimate holds for tubes around any closed submanifold assuming only a pointwise $k$-Ricci lower bound (see also [6] for a related volume comparison using pointwise $k$-Ricci bounds).

Loosely speaking, the estimate of Theorem 1.1 shows that it does not matter how the negative part of the curvature concentrates around the submanifold, a uniform estimate holds for all manifolds with $\left\|\left(\rho_{k}-H\right)_{-}\right\|_{p}$ bounded above by a constant as long as $p$ is chosen sufficiently large. The estimates of Gallot and PetersenWei for tubes around hypersurfaces and geodesic balls require $p>n / 2$, whereas the estimates of Petersen-Shteingold-Wei for the tubes around a geodesic require $p>n-1$. Notice that our requirement $p>n-k$ is a natural generalization of both of these conditions as $n-k$ is bounded below by $n / 2$.

As a simple consequence of Theorem 1.1 we obtain the following uniform lower bound for the volume of closed minimal submanifolds in spaces with integral curvature bounds.

Corollary 1.4. Given integers $n$ and $m$ with $n \geq 3$ and $0<m<n-1$, and real numbers $H \leq 0, v_{0}, D>0$ and $p>n-k$ where $k=\min \{m, n-m-1\}$, there exist constants $\epsilon\left(n, m, p, H, v_{0}, D\right)>0$ and $\delta\left(n, m, p, H, v_{0}, D\right)>0$ such that every closed $n$-dimensional Riemannian manifold $M$ satisfying

$$
\operatorname{vol}(M) \geq v_{0}, \quad \operatorname{diam}(M) \leq D, \quad\left\|\left(\rho_{k}-H\right)_{-}\right\|_{p} \leq \epsilon
$$

has the property that all closed m-dimensional minimal submanifolds have volume bounded below by $\delta$.

Remark 1.5. This should be thought of as a generalization of Cheeger's lemma. For the case of 1-dimensional minimal submanifolds (closed geodesics) this result was obtained already in [12, Theorem 1.2]. The proof follows easily from the observation that our uniform upper bound for the tube around a minimal submanifold approaches 0 as $\operatorname{vol}(\Sigma),\left\|\left(\rho_{k}-H\right)_{-}\right\|_{p} \rightarrow 0$.

Before proving Theorem 1.1 we introduce our main ideas by proving a new Hessian comparison for distance functions based on $k$-Ricci curvature bounds which is of independent interest. Specifically, if $r(x)=d(x, \Sigma)$ is the distance to a closed submanifold $\Sigma$ we prove an upper bound for certain partial traces of the Hessian $\nabla^{2} r$ given pointwise lower bounds on $R i c_{k}$. This Hessian comparison unifies and generalizes a number of distinct Hessian and Laplacian comparisons for the distance function to a point. Recall that in a space of constant curvature $H$ the eigenvalues of the Hessian of the distance function to a point are given by $\operatorname{cs}_{H}(r) / \operatorname{sn}_{H}(r)$ where $\mathrm{sn}_{H}$ and $\mathrm{cs}_{H}$ are the generalized trigonometric functions defined in Section 3.

Theorem 1.6 (Hessian Comparison). Let $\Sigma^{m}$ be an $m$-dimensional submanifold of a complete Riemannian manifold $M^{n}$ and let $r(x)=d(x, \Sigma)$ be the distance function to $\Sigma$. 


$$
\begin{aligned}
& \text { Let } \gamma:\left[0, t_{0}\right) \rightarrow M \text { be any geodesic segment satisfying } r(\gamma(t))=t \text {. If } \\
& \qquad \operatorname{Ric}_{k}(\dot{\gamma}, \cdot) \geq k H
\end{aligned}
$$

for some constant $H$ then for any orthonormal $k$-frame $\left\{e_{1}(t), \ldots, e_{k}(t)\right\} \subset T_{\gamma(t)} M$ which is parallel along $\gamma$ we have

$$
\sum_{i=1}^{k} \nabla^{2} r\left(e_{i}, e_{i}\right) \leq \begin{cases}k \frac{h_{0} \operatorname{cs}_{H}(r)-H \operatorname{sn}_{H}(r)}{\operatorname{cs}_{H}(r)+h_{0} \operatorname{sn}_{H}(r)} & \text { if }\left\{e_{1}(0), \ldots, e_{k}(0)\right\} \subset T \Sigma \\ k \operatorname{cs}_{H}(r) / \operatorname{sn}_{H}(r) & \text { otherwise }\end{cases}
$$

where $h_{0}=\frac{1}{k} \sum_{i=1}^{k}\left\langle S_{\dot{\gamma}(0)}\left(e_{i}\right), e_{i}\right\rangle$ and $S_{\dot{\gamma}(0)}$ is the Weingarten map of $\Sigma$ for the normal $\dot{\gamma}(0)$.

Remark 1.7. Notice that taking $\Sigma$ to be a point, the usual Hessian and Laplacian comparisons follow from this theorem by taking $k=1$ and $k=n-1$, respectively. When $\Sigma$ is a point, the result was proved by Shen [19, Lemma 11] and Li-Wang $[10$, Theorem 1.2].

Remark 1.8. This result implies the mean curvature comparison of [6] when $\Sigma$ is totally geodesic.

In Section 3 we give a slightly more general version of this theorem which also treats the question of rigidity when equality holds. This comparison theorem should be compared with that of Guijarro-Wilhelm which gives comparison along a family of $k$-dimensional subspaces determined by Jacobi fields rather than parallel subspaces [7, Lemma 2.23]. That comparison is based on Wilking's transverse Jacobi equation; by contrast, Theorem 1.6 above is based on the comparison theory for a Riccati differential equation and thus yields an elementary proof of the volume comparison of Section 4.

In Section 4 we also use this Hessian comparison to generalize another HeintzeKarcher type inequality of G. Qiu and C. Xia relating the volume of a compact manifold with boundary to the total inverse mean curvature of the boundary [17, Theorem 1.3]. They originally proved this inequality assuming a lower bound on sectional curvature and ask whether the inequality holds assuming only a Ricci lower bound. In Theorem 4.2 we show that an $(n-2)$-Ricci lower bound suffices.

The paper is structured as follows. In Section 2 we start with a brief review of the basic notions underlying the geometry of volume comparison and fix notation. In Sections 3 and 4 we give a proof of Theorem 1.6 and apply it to obtain several Heintze-Karcher type volume inequalities for $k$-Ricci curvature mentioned above. We conclude in Section 5 with the proof of Theorem 1.1.

Acknowledgements. I would like to express gratitude to my advisor, Guofang Wei, for many helpful discussions and invaluable feedback in the preparation of this paper. I would also like to thank Frederick Wilhelm for several suggestions to help clarify the exposition. The author was supported in part by the National Science Foundation under the grant DMS-1506393.

\section{Preliminaries}

2.1. Polar volume density, mean curvature, and distance. Fix, once and for all, a complete, connected Riemannian manifold $\left(M^{n}, g\right)$ of dimension $n$. Given a closed $m$-dimensional (embedded) submanifold $\Sigma$ of $M$ let $\nu=\nu(\Sigma)$ denote the normal bundle of $\Sigma, \nu_{x}$ the fiber over $x \in \Sigma$, and let $\hat{\nu}$ denote the unit normal 
bundle. The normal bundle has a canonical Riemannian metric such that the projection is a Riemannian submersion and each tangent space splits orthogonally into a vertical subspace tangent to the fiber and a horizontal subspace consisting of vectors tangent to curves in $\nu$ which are parallel along their base curve (with respect to the normal connection).

It is well-known that the exponential map on the normal bundle $\exp _{\nu}: \nu \rightarrow M$ restricts to a diffeomorphism from the open neighborhood

$$
U=\left\{u \in \nu: d\left(\Sigma, \exp _{\nu}((1+\epsilon) u)\right)=|(1+\epsilon) u| \text { for some } \epsilon>0\right\}
$$

of the zero section onto a set $\exp _{\nu}(U)$ such that $M \backslash \exp _{\nu}(U)$ has measure zero (see e.g. $[11,5])$. Define the polar volume density function $\mathcal{A}: \nu \rightarrow \mathbb{R}$ as the density of the volume element of $M$ written in $\Sigma$-polar coordinates on $U$, i.e. for normal vectors $u \in U, \mathcal{A}(u)$ can be defined using the Jacobian determinant of the normal exponential map by

$$
\mathcal{A}(u)=\left|d\left(\exp _{\nu}\right)_{u}\right| \cdot|u|^{n-m-1}
$$

with $\mathcal{A}$ extended to all of $\nu$ by setting $\mathcal{A} \equiv 0$ on $\nu \backslash U$. Here, we have anticipated the use of Fubini's theorem to integrate over the normal bundle as an iterated integral whereupon the volume of the tube $T(\Sigma, r)$ can be written

$$
\operatorname{vol}(T(\Sigma, r))=\int_{0}^{r} \int_{\hat{\nu}} \mathcal{A}(t, \xi) d \xi d t
$$

Above and henceforth we put $\mathcal{A}(t, \xi)=\mathcal{A}(t \xi)$ for $\xi \in \hat{\nu}$. Note that since $\exp _{\nu}$ is an isometry on the zero section of the normal bundle $\mathcal{A}(t, \xi) \sim t^{n-m-1}$ as $t \rightarrow 0$.

Let $r: M \rightarrow \mathbb{R}$ be the distance function from the submanifold $\Sigma$ and let $\Sigma_{t}=$ $r^{-1}(t) \cap \exp _{\nu}(U)$ denote the part of the level set consisting of regular points of $r$. For $u \in U$ with $|u|=t>0$, the gradient $\nabla r$ near $x=\exp _{\nu}(u)$ is a unit normal along $\Sigma_{t}$ and hence the Hessian $\nabla^{2} r$ at $x$ is equivalent to the shape operator of the level set $\Sigma_{t}$ at $x$ denoted

$$
S(t, \xi): T_{x} \Sigma_{t} \rightarrow T_{x} \Sigma_{t} .
$$

The relative rate of change of the polar volume density in the radial direction is precisely the mean curvature $h(t, \xi)=\operatorname{tr}(S(t, \xi))$ of the distance level sets $\Sigma_{t}$; i.e.

$$
\mathcal{A}^{\prime}=h \mathcal{A} \text {. }
$$

Finally, we fix notation concerning the extrinsic geometry of $\Sigma$. For $\xi \in \hat{\nu}$ based at $x \in \Sigma$, let $S_{\xi}: T_{x} \Sigma \rightarrow T_{x} \Sigma$ denote the Weingarten map $(\nabla \xi)^{\top}$ where $\xi$ is extended arbitrarily to a section of $\hat{\nu}$ and ${ }^{\top}$ denotes projection onto $T_{x} \Sigma$. Define the (normalized) mean curvature normal $\eta$ along $\Sigma$ with sign convention chosen so that for all $\xi \in \hat{\nu}$

$$
\langle\eta, \xi\rangle=\frac{\operatorname{tr}\left(S_{\xi}\right)}{m} .
$$

2.2. Evolution equation for the shape operator. For this section we fix $\xi \in \hat{\nu}$ based at $x \in \Sigma$. The one-parameter family of shape operators $S(t)=S(t, \xi)$ satisfies the Riccati differential equation

$$
S^{\prime}+S^{2}=-R_{\partial_{r}}
$$

where $\partial_{r}=\nabla r$ denotes the gradient, $R_{\partial_{r}}$ denotes the directional curvature operator $R_{\partial_{r}}(X)=R\left(X, \partial_{r}\right) \partial_{r}$, and the prime notation denotes the covariant derivative in the direction $\partial_{r}$ (see e.g. [14, Corollary 3.2.10]). 
Of crucial importance in the following analysis are the initial conditions for the matrix Riccati equation above, which depends on the nature of the extension of the family of operators $S(t)$ to $t=0$. In fact, since the square of the distance function is smooth in a neighborhood of $\Sigma$ the scaled family $t S(t)$ extends smoothly through $t=0$. Putting $\gamma(t)=\exp _{\nu}(t \xi)$, if we identify the vector spaces $\dot{\gamma}^{\perp} \subset T_{\gamma(t)} M$ via parallel transport along $\gamma$ with the single vector space $E=\dot{\gamma}(0)^{\perp}$ then expanding in a Taylor series one obtains

$$
S(t)=\frac{1}{t} P_{\xi}+S_{\xi}+O(t)
$$

where $P_{\xi}: E \rightarrow E$ denotes the orthogonal projection onto $\nu_{x} \cap \xi^{\perp}$ and $S_{\xi}$ is the Weingarten map extended trivially to the orthogonal complement of $T_{x} \Sigma$ in $E$.

\section{Hessian Comparison}

Using the notation from Section 2, we now state and prove a more complete version of the Hessian comparison theorem given in the introduction. The idea is that we can control certain partial traces of the Hessian of a distance function using the $k$-Ricci curvature. For a linear operator $T$ on a real inner product space $\mathcal{V}$, and a $k$-dimensional subspace $\mathcal{W} \subset \mathcal{V}$, the partial trace of $T$ on $\mathcal{W}$ is defined by

$$
\operatorname{tr}_{\mathcal{W}}(T)=\sum_{i=1}^{k}\left\langle T\left(e_{i}\right), e_{i}\right\rangle
$$

where $\left\{e_{1}, \ldots, e_{k}\right\}$ is any orthonormal basis of $\mathcal{W}$.

Define the generalized trigonometric functions $\operatorname{sn}_{H}$ and $\operatorname{cs}_{H}$ by

$$
\operatorname{sn}_{H}(r)= \begin{cases}\frac{1}{\sqrt{H}} \sin (\sqrt{H} r) & H>0 \\ r & H=0 \\ \frac{1}{\sqrt{-H}} \sinh (\sqrt{-H} r) & H<0\end{cases}
$$

and $\operatorname{cs}_{H}(r)=\operatorname{sn}_{H}^{\prime}(r)$.

Lemma 3.1 (Hessian Comparison). Let $\Sigma^{m}$ be an $m$-dimensional submanifold of a complete Riemannian manifold $M^{n}$. Fix any $\xi \in \hat{\nu}(\Sigma)$ and put $\gamma(t)=\exp _{\nu}(t \xi)$. Let $\mathcal{W}_{0}$ be any $k$-dimensional subspace of $\xi^{\perp}$ and let $\mathcal{W}_{t} \subset \dot{\gamma}(t)^{\perp}$ denote its parallel translation along $\gamma$. If

$$
\operatorname{Ric}_{k}\left(\dot{\gamma}, \mathcal{W}_{t}\right) \geq k H
$$

for some constant $H$, then for $t$ less than the focal distance in the direction $\xi$, we have

$$
\operatorname{tr}_{\mathcal{W}_{t}}(S(t, \xi)) \leq\left\{\begin{array}{lc}
k \log \left(\operatorname{cs}_{H}(t)+w_{0} \operatorname{sn}_{H}(t)\right)^{\prime} & \text { if } \mathcal{W}_{0} \subset T \Sigma \\
k \log \left(\operatorname{sn}_{H}(t)\right)^{\prime} & \text { otherwise }
\end{array}\right.
$$

where $w_{0}=\operatorname{tr}_{\mathcal{W}_{0}}\left(S_{\xi}\right) / k$. If equality holds at $t_{0}$, then equality holds on $\left(0, t_{0}\right]$ and

(1) $H$ is an eigenvalue of $R_{\dot{\gamma}}$ with $\mathcal{W}_{t_{0}}$ contained in the corresponding eigenspace,

(2) $\mathcal{W}_{t_{0}}^{\perp}$ is an invariant subspace of $R_{\dot{\gamma}}$, and

(3) either $\mathcal{W}_{0} \subset \nu$ or $\mathcal{W}_{0}$ is contained in an eigenspace of $S_{\xi}$.

Proof. Using the fact that $\mathcal{W}_{t}$ is parallel along $\gamma$, we may choose a parallel orthonormal basis $\left\{e_{1}, \ldots, e_{n-1}\right\}$ for $\dot{\gamma}^{\perp}$ such that $\left\{e_{1}, \ldots, e_{k}\right\}$ form a parallel orthonormal basis of $\mathcal{W}_{t}$. For $0<t<t_{f}$, where $t_{f}$ is the focal distance along $\gamma$, the shape operators $S(t)=S(t, \xi)$ satisfy the Riccati equation $(2.3)$, and since $\mathcal{W}_{t}$ is parallel 
along $\gamma$ the partial trace $\operatorname{tr}_{\mathcal{W}_{t}}$ commutes with the covariant derivative along $\gamma$ and hence

$$
\operatorname{tr}_{\mathcal{W}_{t}}(S)^{\prime}+\operatorname{tr}_{\mathcal{W}_{t}}\left(S^{2}\right)=-\operatorname{Ric}_{k}\left(\dot{\gamma}, \mathcal{W}_{t}\right)
$$

Putting $s_{i j}=\left\langle S e_{i}, e_{j}\right\rangle$ and using the symmetry $s_{i j}=s_{j i}$ of the Hessian we have

$$
\operatorname{tr}_{\mathcal{W}_{t}}\left(S^{2}\right)=\sum_{i=1}^{k} \sum_{j=1}^{n-1} s_{i j}^{2} \geq \sum_{i, j=1}^{k} s_{i j}^{2} \geq \sum_{i=1}^{k} s_{i i}^{2} \geq \frac{1}{k}\left(\sum_{i=1}^{k} s_{i i}\right)^{2}=\frac{1}{k} \operatorname{tr}_{\mathcal{W}_{t}}(S)^{2}
$$

where the last inequality follows from the Cauchy-Schwarz inequality. Putting $w(t)=\operatorname{tr}_{\mathcal{W}_{t}}(S(t)) / k$ we have

$$
w^{\prime}(t)+w(t)^{2} \leq-\operatorname{Ric}_{k}\left(\dot{\gamma}, \mathcal{W}_{t}\right) / k \leq-H .
$$

Noting that the model functions on the right hand of equation (3.1) satisfy the Riccati equation $(f / k)^{\prime}+(f / k)^{2}=-H$, we may apply the comparison theory for this equation provided we match the initial conditions. Using the Taylor expansion (2.4) we see that if $\mathcal{W}_{0} \subset T_{\gamma(0)} \Sigma$ then $w(t) \rightarrow w_{0}$ as $t \rightarrow 0$, and otherwise $w(t)=$ $O\left(t^{-1}\right)$ as $t \rightarrow 0$. The inequality now follows from the comparison theory for the scalar Riccati equation (see e.g. [14, Proposition 6.4.1]).

If equality holds for some $t_{0} \leq t_{f}$, then the aforementioned Riccati comparison principle implies that equality holds on $\left(0, t_{0}\right]$. From the inequalities above, it follows that with respect to the parallel basis $\left\{e_{i}\right\}$ the matrix representation of $S(t)$ on $\left(0, t_{0}\right]$ is block diagonal of the form

$$
S(t)=\left(\begin{array}{cc}
w(t) I_{k} & 0 \\
0 & *
\end{array}\right)
$$

where $I_{k}$ is the $k \times k$ identity matrix. Since this decomposition holds on $\left(0, t_{0}\right]$, it follows from the expansion (2.4) that either $\mathcal{W}_{0} \subset T \Sigma$ or $\mathcal{W}_{0} \subset \nu$. Moreover, if $\mathcal{W}_{0} \subset T \Sigma$ then (2.4) implies that $\mathcal{W}_{0}$ is contained in an eigenspace of $S_{\xi}$ with eigenvalue $w_{0}=\lim _{t \rightarrow 0} w(t)$. Parts (1) and (2) then follow from the observation that $-S^{\prime}-S^{2}=R_{\dot{\gamma}}$ is also block diagonal of the same form, replacing $w(t)$ with $-w^{\prime}-w^{2}=H$.

From the proof above it is easily seen that equality is realized if $M$ is a space of constant curvature $H$ and $\Sigma$ is a submanifold such that condition (3) in the lemma holds for $\Sigma$ and $\mathcal{W}_{t}$.

Our main applications of this lemma will be to the volume inequalities of the next section; however, we also recover an upper bound on the focal radius which was recently obtained by Guijarro and Wilhelm using a different Jacobi field comparison for $k$-Ricci curvature [7].

Corollary 3.2. Let $\Sigma$ be a submanifold of a complete Riemannian manifold $M^{n}$ with $\operatorname{dim}(\Sigma) \geq k$. If Ric $c_{k} \geq k \cdot H>0$ then the focal radius of $\Sigma$ is at most $\frac{\pi}{2 \sqrt{H}}$ and this focal radius is achieved if and only if $\Sigma$ is totally geodesic.

Proof of Corollary 3.2. Given $x \in \Sigma$ let $\xi \in \hat{\nu}(\Sigma)$ be any unit normal based at $x$, and put $\gamma(t)=\exp _{\nu}(t \xi)$. By replacing $\xi$ with $-\xi$ if necessary, we may assume $\langle\eta, \xi\rangle \leq 0$ where $\eta$ is the mean curvature vector of $\Sigma$. Putting $\mathcal{W}_{0}=T_{x} \Sigma$ and applying the lemma, we find that $\operatorname{tr}_{\mathcal{W}_{t}}(S(t, \xi))$ diverges to $-\infty$ for some $t \leq \pi /(2 \sqrt{H})$. Moreover, if equality holds for all $\xi \in \hat{\nu}(\Sigma)$ then $\eta \equiv 0$ and it follows from part (3) of the lemma that $\Sigma$ is totally umbilic, and hence totally geodesic. 


\section{Volume inequalities USing $k$-Ricci CURVATURe Bounds}

Using the Hessian comparison above we prove two different Heintze-Karcher type volume inequalities using $k$-Ricci lower bounds.

Theorem 4.1. Let $M^{n}$ be a complete Riemannian manifold and let $\Sigma^{m}$ be a closed $m$-dimensional submanifold. Put $k=\min \{m, n-m-1\}$. If Ric $c_{k} \geq k \cdot H$ then

$$
\operatorname{vol}(T(\Sigma, r)) \leq \int_{\hat{\nu}} \int_{0}^{z(r, \xi)}\left(\operatorname{cs}_{H}(t)+\langle\eta, \xi\rangle \operatorname{sn}_{H}(t)\right)^{m} \operatorname{sn}_{H}(t)^{n-m-1} d t d \xi .
$$

where $z(r, \xi)$ denotes the minimum of $r$ and the first zero of the integrand.

Proof. Given $\xi \in \hat{\nu}(\Sigma)$ based at $x \in \Sigma$ let $\gamma(t)=\exp _{\nu}(t \xi)$ and let $\mathcal{H}_{t}$ and $\mathcal{V}_{t}$ denote the subspaces of $\dot{\gamma}(t)^{\perp}$ parallel along $\gamma$ to $T_{x} \Sigma$ and $\nu_{x}(\Sigma)$, respectively. Since $\mathcal{H}_{t}$ and $\mathcal{V}_{t}$ are orthogonal, the mean curvature $h(t, \xi)$ of $\Sigma_{t}$ at $\gamma(t)$ is given by

$$
h=\varphi+\psi
$$

where $\varphi=\operatorname{tr}_{\mathcal{H}_{t}}(S(t, \xi))$ and $\psi=\operatorname{tr}_{\mathcal{V}_{t}}(S(t, \xi))$. Using the assumption Ric $_{k} \geq k H$, Lemma 3.1 gives

$$
h(t, \xi) \leq m \log \left(\operatorname{cs}_{H}(t)+\langle\eta, \xi\rangle \operatorname{sn}_{H}(t)\right)^{\prime}+(n-m-1) \log \left(\operatorname{sn}_{H}(t)\right)^{\prime}
$$

for $0<t<t_{c}(\xi)$ where $t_{c}(\xi)$ is the distance to the cut locus of $\Sigma$ in the direction $\xi$. From equation $(2.2)$ we have for $0<t<t_{c}$ the identity

$$
\log (\mathcal{A})^{\prime}=\log \left[\left(\operatorname{cs}_{H}+\langle\eta, \xi\rangle \operatorname{sn}_{H}\right)^{m} \operatorname{sn}_{H}^{n-m-1}\right]^{\prime} .
$$

Now, since $\mathcal{A}(t, \xi) \sim t^{n-m-1}$ as $t \rightarrow 0$ we can integrate from 0 to $t \leq t_{c}$ to obtain

$$
\mathcal{A}(t, \xi) \leq\left(\mathrm{cs}_{H}+\langle\eta, \xi\rangle \operatorname{sn}_{H}\right)^{m} \operatorname{sn}_{H}^{n-m-1}
$$

The result follows from equation (2.1).

Our second application of the Hessian comparison is a generalization of another Heintze-Karcher type inequality given by G. Qiu and C. Xia in [17]. The inequality of Qiu-Xia is motivated by similar inequalities of A. Ros and S. Brendle which have been used to prove Alexandrov's Theorem in various contexts (see $[18,3,17]$ ).

Theorem 4.2. Let $\left(M^{n}, g\right)$ be a compact Riemannian manifold with smooth boundary $\Sigma$ with outward unit normal $\xi$ and mean curvature vector $\eta$. Fix a point $p \in M$ and put $f(x)=\cosh (r(x))$ where $r(x)=d(x, p)$ is the distance from $x$ to $p$ in $M$.

If $\langle\eta, \xi\rangle>0$ everywhere on $\Sigma$ and Ric $_{n-2} \geq-(n-2)$ then

$$
\int_{\Sigma} \frac{f}{\langle\eta, \xi\rangle} \operatorname{dvol}_{\Sigma} \geq \int_{M}(\Delta f) \operatorname{dvol}_{M}
$$

Equality holds if and only if $M$ is a geodesic ball in a space form of constant sectional curvature -1 .

Remark 4.3. This theorem was proved in [17] using the assumption Ric $_{1} \geq-1$ (i.e. sec $\geq-1)$ in place of $R i c_{n-2} \geq-(n-2)$. The theorem above shows that an $(n-2)$-Ricci lower bound suffices.

The proof is based on the following weighted version of Reilly's formula. 
Theorem 4.4 (Qiu-Xia, 2014). Let $\left(M^{n}, g\right)$ be a compact Riemannian manifold with smooth boundary $\Sigma$ and outward unit normal $\xi$ and let $f: M \rightarrow \mathbb{R}$ be a.e. twice differentiable. Given $u \in C^{\infty}(M)$ and $\lambda \in \mathbb{R}$ such that $\left.u\right|_{\Sigma}=u_{0}$ is constant

$$
\begin{aligned}
(n-1) \int_{\Sigma} f & \langle\eta, \xi\rangle\langle\nabla u, \xi\rangle^{2}+2 \lambda f\langle\nabla u, \xi\rangle u_{0}-\langle\nabla f, \xi\rangle \lambda u_{0}^{2} d v o l_{\Sigma} \\
\quad & =\int_{M} f\left((\Delta u+n \lambda u)^{2}-\left|\nabla^{2} u+\lambda u g\right|^{2}\right)-(n-1) \lambda(\Delta f+n \lambda f) u^{2} d v o l_{M} \\
& +\int_{M}\left(\Delta f g-\nabla^{2} f-f R i c+2(n-1) \lambda f g\right)(\nabla u, \nabla u) \text { dvol }_{M}
\end{aligned}
$$

where $\eta$ is the mean curvature vector of $\Sigma$.

Proof of Theorem 4.2. First observe that

$$
\nabla^{2} f=\cosh (r) d r^{2}+\sinh (r) \nabla^{2} r .
$$

For any $x$ outside the cut locus of $p$ the Hessian $\nabla^{2} f$ thus has an orthonormal frame of eigenvectors $\left\{e_{1}, \ldots, e_{n-1}, \nabla r\right\}$ at $x$ with dual frame $\left\{\theta^{1}, \ldots, \theta^{n-1}, d r\right\}$ so that

$$
\nabla^{2} f=\cosh (r) d r^{2}+\sinh (r) \sum_{i=1}^{n-1} \kappa_{i}\left(\theta^{i}\right)^{2}
$$

where $\kappa_{i}=\nabla^{2} r\left(e_{i}, e_{i}\right)$. It follows that

$$
(\Delta f) g-\nabla^{2} f=\sinh (r)(\Delta r) d r^{2}+\sum_{i=1}^{n-1}\left(\cosh (r)+\sinh (r) \sum_{j \neq i} \kappa_{j}\right)\left(\theta^{i}\right)^{2} .
$$

Using the assumption $\operatorname{Ric}_{n-2} \geq-(n-2)$, the Hessian comparison theorem above then implies that $\Delta r \leq(n-1) \cosh (r) / \sinh (r)$ and $\sum_{j \neq i} \kappa_{j} \leq(n-2) \cosh (r) / \sinh (r)$ and hence

$$
(\Delta f) g-\nabla^{2} f \leq(n-1) f g .
$$

The rest of the proof is the same as that in [17], but we include it for completeness. Put $\lambda=-1$ and let $u$ be the solution to the Dirichlet boundary value problem

$$
\left\{\begin{array}{l}
\Delta u=n u \\
\left.u\right|_{\Sigma}=c>0
\end{array}\right.
$$

From equation (4.2) it follows that

$$
\left(\Delta f g-\nabla^{2} f-f R i c-2(n-1) f g\right)(\nabla u, \nabla u) \leq 0
$$

and

$$
(n-1)(\Delta f-n f) u^{2} \leq 0
$$

and hence the formula of Theorem 4.4 gives

$$
\int_{\Sigma} f\langle\eta, \xi\rangle\langle\nabla u, \xi\rangle^{2} \leq \int_{\Sigma}\left(2 f\langle\nabla u, \xi\rangle c-\langle\nabla f, \xi\rangle c^{2}\right) .
$$

Now, using Hölder's inequality followed by the previous inequality we get

$$
\begin{aligned}
\left(\int_{\Sigma} f\langle\nabla u, \xi\rangle\right)^{2} & \leq \int_{\Sigma} \frac{f}{\langle\eta, \xi\rangle} \int_{\Sigma} f\langle\eta, \xi\rangle\langle\nabla u, \xi\rangle^{2} \\
& \leq \int_{\Sigma} \frac{f}{\langle\eta, \xi\rangle} \int_{\Sigma}\left(2 f\langle\nabla u, \xi\rangle c-\langle\nabla f, \xi\rangle c^{2}\right) .
\end{aligned}
$$


It follows that

$$
\left(\int_{\Sigma} f\langle\nabla u, \xi\rangle-c \int_{\Sigma} \frac{f}{\langle\eta, \xi\rangle}\right)^{2}-c^{2}\left(\int_{\Sigma} \frac{f}{\langle\eta, \xi\rangle}\right)^{2} \leq-c^{2} \int_{\Sigma} \frac{f}{\langle\eta, \xi\rangle} \int_{\Sigma}\langle\nabla f, \xi\rangle .
$$

Dropping the first term (since it's nonnegative) and noting that $c \neq 0$ we obtain

$$
\int_{\Sigma} \frac{f}{\langle\eta, \xi\rangle} \geq \int_{\Sigma}\langle\nabla f, \xi\rangle=\int_{M} \Delta f
$$

as desired. If equality holds than equality must hold in equation 4.3 and hence $(\Delta u-n u)^{2}=\left|\nabla^{2} u-u g\right|^{2}$. By construction $\Delta u=n u$ and hence in the equality case we have $\nabla^{2} u=u g$ in $M$. Since $\left.u\right|_{\Sigma}=c$ it follows from an Obata type rigidity result that $M$ must be a geodesic ball in a space form of constant curvature -1 (see e.g. [22, Theorem 5.1]).

\section{Volume estimates Using INTEGRAL CURVATURE BOUNDS}

We now give the proof of Theorem 1.1. In particular, throughout this section we assume $0<m<n-1$. In the proof of Theorem 4.1 we assumed a pointwise lower curvature bound and used the partial traces of the Riccati equation (2.3) to bound the mean curvature $h$ explicitly and thus bound the logarithmic growth of the volume density $\mathcal{A}$. Unfortunately, in order to obtain a bound depending only on integrals of the curvature this approach fails since one cannot use the comparison theory for the Riccati differential equation.

As before, we fix $\xi \in \hat{\nu}$ and put $\gamma(t)=\exp _{\nu}(t \xi)$. Differentiating eq. (2.2) gives

$$
\mathcal{A}^{\prime \prime}=\left(h^{\prime}+h^{2}\right) \mathcal{A} \text {. }
$$

Taking the trace of equation $(2.3)$ gives $h^{\prime}+\operatorname{tr}\left(S^{2}\right)=-\operatorname{Ric}(\dot{\gamma}, \dot{\gamma})$ which leaves us to control the second order invariant $\operatorname{tr}(S)^{2}-\operatorname{tr}\left(S^{2}\right)$ in (5.1) in terms of curvature. This motivates us $[4,25]$ to consider in place of $\mathcal{A}$ the function $A$ with $\mathcal{A}=A^{n-1}$ which satisfies

$$
A^{\prime \prime}=\frac{1}{n-1}\left(h^{\prime}+\frac{h^{2}}{n-1}\right) A \leq-\frac{\operatorname{Ric}(\dot{\gamma}, \dot{\gamma})}{n-1} A
$$

allowing us to control the second order invariant of $S$ using the Cauchy-Schwarz inequality. However, if $0<m<n-1$ then the initial conditions for the function $A$ are unusable, namely $A(0)=0$ and $A^{\prime}(0)=\infty$.

The remarkable observation of [12] was that one can control certain products of eigenvalues of $S$ directly in terms of integrals of sectional curvature without relying on the Cauchy-Schwarz inequality, provided one of the eigenvalues vanishes at $\Sigma$. For $m=1$, putting $\mathcal{A}=A^{n-2}$ (so that $A^{\prime}(0)=1$ ) and assuming $\Sigma$ is a geodesic then allows control of the second order invariant of $S$. However, generalizing this directly to higher dimensional submanifolds by setting $\mathcal{A}=A^{n-m-1}$ then yields an estimate only when $\Sigma$ is totally geodesic.

Instead, motivated by the pointwise comparison of the previous section, we decompose the mean curvature as $h=\varphi+\psi$ as in the proof of Theorem 4.1, and then decompose the polar volume density $\mathcal{A}$ into two functions $\mathcal{A}(t)=\mathcal{J}(t) \mathcal{Y}(t)$ where $\mathcal{J}(t)$ is defined by the equation

$$
\left\{\begin{array}{l}
\mathcal{J}^{\prime}=\varphi \mathcal{J} \\
\mathcal{J}(0)=1
\end{array}\right.
$$


from which it follows that $\mathcal{Y}$ satisfies $\mathcal{Y}^{\prime}=\psi \mathcal{Y}$. Putting $\mathcal{J}=J^{m}$ and $\mathcal{Y}=Y^{n-m-1}$ we have $\mathcal{A}=J^{m} Y^{n-m-1}$ with

$$
J^{\prime \prime}=\frac{1}{m}\left(\varphi^{\prime}+\frac{\varphi^{2}}{m}\right) J \leq-\frac{R i c_{m}\left(\dot{\gamma}, \mathcal{H}_{t}\right)}{m} J
$$

and

$$
Y^{\prime \prime}=\frac{1}{n-m-1}\left(\psi^{\prime}+\frac{\psi^{2}}{n-m-1}\right) Y \leq-\frac{R i c_{n-m-1}\left(\dot{\gamma}, \mathcal{V}_{t}\right)}{n-m-1} Y .
$$

The initial conditions for $J$ and $Y$ are easily found to be

$$
\left\{\begin{array} { l } 
{ J ( 0 ) = 1 , } \\
{ J ^ { \prime } ( 0 ) = \langle \eta , \xi \rangle , }
\end{array} \quad \left\{\begin{array}{l}
Y(0)=0 \\
Y^{\prime}(0)=1
\end{array}\right.\right.
$$

The main challenge introduced with this decomposition is that we only want to consider expressions involving curvature multiplied by the full volume density $\mathcal{A}$, rather than curvature multiplied by just the function $J$ or $Y$ as in (5.2) and (5.3). With this in mind, rather than integrating the two inequalities directly these considerations motivate the following lemma.

Lemma 5.1. If $0<m<n-1$ the functions $J$ and $Y$ defined above satisfy

$$
J^{\prime}(t) Y^{\frac{n-m-1}{m}}(t) \leq \int_{0}^{t}\left(\rho_{m}\right)_{-} \mathcal{A}^{1 / m} d s+\frac{1}{m^{2}} \int_{0}^{t}\left(\varphi_{+} \psi_{+}\right) \mathcal{A}^{1 / m} d s
$$

and

$$
Y^{\prime}(t) J^{\frac{m}{n-m-1}}(t) \leq 1+\int_{0}^{t}\left(\rho_{n-m-1}\right)_{-} \mathcal{A}^{\frac{1}{n-m-1}} d s+\frac{1}{(n-m-1)^{2}} \int_{0}^{t}\left(\varphi_{+} \psi_{+}\right) \mathcal{A}^{\frac{1}{n-m-1}} d s .
$$

Proof. For the first inequality, for any $\delta>0$ we have

$$
\left(J^{\prime} Y^{\delta}\right)^{\prime}=J^{\prime \prime} Y^{\delta}+\delta Y^{\delta-1} J^{\prime} Y^{\prime} .
$$

Using the identities $J^{\prime}=(\varphi / m) J$ and $Y^{\prime}=[\psi /(n-m-1)] Y$ together with eqs. (5.2) and (5.3) we get

$$
\left(J^{\prime} Y^{\delta}\right)^{\prime} \leq\left(-\rho_{m}+\delta \frac{\varphi \psi}{m(n-m-1)}\right) J Y^{\delta} .
$$

Now, if $J^{\prime}(t) \leq 0$ the inequality (5.4) holds automatically so we only need to show the inequality for all values of $t$ such that $J^{\prime}(t)>0$. Moreover, since $J^{\prime}(0) Y^{\delta}(0)=0$ it follows that all such values of $t$ are contained in an interval $\left[t_{0}, t\right]$ such that $J^{\prime}\left(t_{0}\right) Y^{\delta}\left(t_{0}\right)=0$ and $J^{\prime} \geq 0$ on $\left[t_{0}, t\right]$. On such an interval, $J^{\prime} \geq 0$ implies $\varphi \geq 0$ and hence $\varphi \psi \leq \varphi_{+} \psi_{+}$on $\left[t_{0}, t\right]$. Using also that $-\rho_{m} \leq\left(\rho_{m}\right)_{-}$and integrating over the interval $\left[t_{0}, t\right]$ gives

$$
J^{\prime}(t) Y^{\delta}(t) \leq \int_{t_{0}}^{t}\left(\rho_{m}\right)_{-} J Y^{\delta}+\delta \int_{t_{0}}^{t} \frac{\varphi_{+} \psi_{+}}{m(n-m-1)} J Y^{\delta} .
$$

Since the integrands are nonnegative, we can replace the lower bound $t_{0}$ with 0 and preserve the inequality. Finally, taking $\delta=(n-m-1) / m$ gives the result (5.4).

Analogous reasoning leads to the second inequality, except that the initial condition $Y^{\prime}(0) J^{\delta}(0)=1$ leads to the extra term on the right hand side of the inequality.

Based on this lemma, one now only needs to control the product $\varphi_{+} \psi_{+}$in terms of curvature. We prove that this is possible provided $\varphi_{+}$vanishes at $\Sigma$, generalizing the eigenvalue estimate in [12]. 
Lemma 5.2. Let $\varphi, \psi$, and $\mathcal{A}$ be as above. Put $k=\min \{m, n-m-1\}$. If $\varphi_{+}(t) \psi_{+}(t)$ is bounded as $t \rightarrow 0$ then for any $p>n-k$,

$$
\left(\int_{0}^{t}\left(\varphi_{+} \psi_{+}\right)^{p} \mathcal{A} d s\right)^{\frac{1}{p}} \leq \frac{2 p-1}{p-(n-k)}\left(\int_{0}^{t}\left(\rho_{k}\right)_{-}^{p} \mathcal{A} d s\right)^{\frac{1}{p}} .
$$

The proof is given at the end of this section and is a straightforward modification of the proof in [12]. Note that if the submanifold $\Sigma$ is minimal then it follows from equation (2.4) that $\varphi_{+} \psi_{+}$is bounded as $t \rightarrow 0$.

Using these inequalities, we now consider the area of the equidistant hypersurfaces $v(t)=\operatorname{vol}\left(\Sigma_{t}\right)$ given by the integral

$$
v(t)=\int_{\hat{\nu}} J^{m}(t, \xi) Y^{n-m-1}(t, \xi) d \xi .
$$

The volume $V(r)=\operatorname{vol}(T(\Sigma, r))$ can then be written

$$
V(r)=\int_{0}^{r} v(t) d t
$$

We wish to differentiate $v(t)$ via the expression (5.5). Note that the integrand is smooth and nonnegative on the open set $U$ defined in Section 2 and vanishes on $\nu \backslash U$, but may be discontinuous on the boundary of $U$. However, since $U$ is star-shaped with respect to the zero section of $\nu$ (i.e. $u \in U$ implies $\lambda u \in U$ for $0 \leq \lambda \leq 1$ ) it follows that $v$ is an almost everywhere differentiable lower semi-continuous function (see [1]) and

$$
v^{\prime}(t) \leq \int_{\hat{\nu}} m J^{m-1} Y^{n-m-1} J^{\prime}+(n-m-1) J^{m} Y^{n-m-2} Y^{\prime} d \xi .
$$

We now substitute the two inequalities of Lemma 5.1 and use two applications of Hölder's inequality. For example, the first term satisfies

$$
\begin{gathered}
m \int_{\hat{\nu}} J^{m-1} Y^{n-m-1} J^{\prime} d \xi \leq m \int_{\hat{\nu}} \mathcal{A}^{\frac{m-1}{m}}\left(\int_{0}^{t}\left(\rho_{m}\right)_{-} \mathcal{A}^{\frac{1}{m}} d s+\frac{1}{m^{2}} \int_{0}^{t}\left(\varphi_{+} \psi_{+}\right) \mathcal{A}^{\frac{1}{m}} d s\right) d \xi \\
\leq m v(t)^{\frac{m-1}{m}}\left[\left(\int_{\hat{\nu}}\left(\int_{0}^{t}\left(\rho_{m}\right)_{-} \mathcal{A}^{\frac{1}{m}} d s\right)^{m} d \xi\right)^{\frac{1}{m}}+\frac{1}{m^{2}}\left(\int_{\hat{\nu}}\left(\int_{0}^{t}\left(\varphi_{+} \psi_{+}\right) \mathcal{A}^{\frac{1}{m}} d s\right)^{m} d \xi\right)^{\frac{1}{m}}\right] \\
\quad \leq m v(t)^{\frac{m-1}{m}} t^{\frac{m-1}{m}}\left[\left(\int_{\hat{\nu}} \int_{0}^{t}\left(\rho_{m}\right)_{-}^{m} \mathcal{A} d s d \xi\right)^{\frac{1}{m}}+\frac{1}{m^{2}}\left(\int_{\hat{\nu}} \int_{0}^{t}\left(\varphi_{+} \psi_{+}\right)^{m} \mathcal{A} d s d \xi\right)^{\frac{1}{m}}\right] .
\end{gathered}
$$

Handling the second term of the integral in a similar fashion one easily checks that

$$
\begin{aligned}
v^{\prime}(t) & \leq(n-m-1) \operatorname{vol}(\hat{\nu})^{\frac{1}{n-m-1}} v(t)^{\frac{n-m-2}{n-m-1}} \\
& +\left[(n-m-1)\left\|\left(\rho_{n-m-1}\right)_{-}\right\|_{n-m-1, t}+\frac{1}{n-m-1}\left\|\varphi_{+} \psi_{+}\right\|_{n-m-1, t}\right](t v(t))^{\frac{n-m-2}{n-m-1}} \\
& +\left[m\left\|\left(\rho_{m}\right)_{-}\right\|_{m, t}+\frac{1}{m}\left\|\varphi_{+} \psi_{+}\right\|_{m, t}\right](t v(t))^{\frac{m-1}{m}}
\end{aligned}
$$

where $\|\cdot\|_{p, t}$ is the usual $L^{p}$ norm on the tube $T(\Sigma, t)$. In order to make use of the estimate in Lemma 5.2 it is necessary to raise the exponents in the expression above at the cost of a volume term via the inequality

$$
\|f\|_{q, t} \leq\|f\|_{p, t} V(t)^{\frac{1}{q}-\frac{1}{p}}
$$

provided $p \geq q \geq 1$. 
Henceforth, set $k=\min \{m, n-m-1\}$ and note that $\rho_{k} \leq \rho_{n-k-1}$. Using the inequality (5.7) together with Lemma 5.2, we have for any $p>n-k \geq q$

$$
\left\|\varphi_{+} \psi_{+}\right\|_{q, t} \leq \frac{2 p-1}{p-(n-k)} V(t)^{\frac{1}{q}-\frac{1}{p}}\left\|\left(\rho_{k}\right)_{-}\right\|_{p, t}
$$

Applying these observations to (5.6) we obtain

$$
\begin{aligned}
V^{\prime \prime}(t) \leq & (n-m-1) \operatorname{vol}(\hat{\nu})^{\frac{1}{n-m-1}} V^{\prime}(t)^{\frac{n-m-2}{n-m-1}} \\
& +\left\|\left(\rho_{k}\right)_{-}\right\|_{p, t}\left(C_{1} V(t)^{\frac{1}{m}-\frac{1}{p}}\left(t V^{\prime}(t)\right)^{\frac{m-1}{m}}+C_{2} V(t)^{\frac{1}{n-m-1}-\frac{1}{p}}\left(t V^{\prime}(t)\right)^{\frac{n-m-2}{n-m-1}}\right)
\end{aligned}
$$

where

$$
\begin{aligned}
& C_{1}=m+\frac{2 p-1}{m(p-n+k)}, \\
& C_{2}=(n-m-1)+\frac{2 p-1}{(n-m-1)(p-n+k)} .
\end{aligned}
$$

In order to obtain an inequality which depends more generally on $\left\|\left(\rho_{k}-H\right)_{-}\right\|$we observe that for $H \leq 0$,

$$
\left(\rho_{k}\right)_{-} \leq\left(\rho_{k}-H\right)_{-}+|H|
$$

and hence $\left(\rho_{k}\right)_{-}^{p} \leq 2^{p-1}\left(\left(\rho_{k}-H\right)_{-}^{p}+|H|^{p}\right)$. It then follows that

$$
\left\|\left(\rho_{k}\right)_{-}\right\|_{p, t} \leq 2^{\frac{p-1}{p}}\left(\left\|\left(\rho_{k}-H\right)_{-}\right\|_{p, t}+|H| V(t)^{\frac{1}{p}}\right) .
$$

Substituting this back into the inequality above and estimating $2^{\frac{p-1}{p}}<2$ we obtain

$$
\begin{aligned}
V^{\prime \prime}(t) \leq & (n-m-1) \operatorname{vol}(\hat{\nu})^{\frac{1}{n-m-1}} V^{\prime}(t)^{\frac{n-m-2}{n-m-1}} \\
& +2\left\|\left(\rho_{k}-H\right)_{-}\right\|_{p}\left(C_{1} V(t)^{\frac{1}{m}-\frac{1}{p}}\left(t V^{\prime}(t)\right)^{\frac{m-1}{m}}+C_{2} V(t)^{\frac{1}{n-m-1}-\frac{1}{p}}\left(t V^{\prime}(t)\right)^{\frac{n-m-2}{n-m-1}}\right) \\
& +2|H|\left(C_{1} V(t)^{\frac{1}{m}}\left(t V^{\prime}(t)\right)^{\frac{m-1}{m}}+C_{2} V(t)^{\frac{1}{n-m-1}}\left(t V^{\prime}(t)\right)^{\frac{n-m-2}{n-m-1}}\right) .
\end{aligned}
$$

It remains to use this differential inequality to obtain an estimate for $V(t)$. To simplify notation we introduce the constants

$$
\begin{aligned}
a & =(n-m-1)\left(\operatorname{vol}\left(\mathbb{S}^{n-m-1}\right) \operatorname{vol}(\Sigma)\right)^{\frac{1}{n-m-1}}, \\
b & =\left\|\left(\rho_{k}-H\right)_{-}\right\|_{p} \\
c & =2(n-k-1)+\frac{2}{k}\left(\frac{2 p-1}{p-n+k}\right) .
\end{aligned}
$$

Noting that $\operatorname{vol}(\hat{\nu})=\operatorname{vol}\left(\mathbb{S}^{n-m-1}\right) \operatorname{vol}(\Sigma)$ the previous inequality then implies

$$
\begin{aligned}
V^{\prime \prime}(t) \leq & a\left(V^{\prime}\right)^{1-\frac{1}{n-m-1}}+c b\left(V^{\frac{1}{m}-\frac{1}{p}}\left(t V^{\prime}\right)^{1-\frac{1}{m}}+V^{\frac{1}{n-m-1}-\frac{1}{p}}\left(t V^{\prime}\right)^{1-\frac{1}{n-m-1}}\right) \\
& +c|H|\left(V(t)^{\frac{1}{m}}\left(t V^{\prime}(t)\right)^{1-\frac{1}{m}}+V(t)^{\frac{1}{n-m-1}}\left(t V^{\prime}(t)\right)^{1-\frac{1}{n-m-1}}\right) .
\end{aligned}
$$

Multiplying through by the nonnegative quantity $\left(V^{\prime}\right)^{\frac{1}{n-k-1}}$ and putting

$$
\delta_{1}=\frac{1}{n-m-1}-\frac{1}{n-k-1}, \quad \delta_{2}=\frac{1}{k}-\frac{1}{n-k-1}, \quad \delta_{3}=\frac{1}{n-k-1}-\frac{1}{p},
$$

and $\alpha=(n-k-1) /(n-k)$ gives

$$
\begin{aligned}
V^{\prime \prime}(t)\left(V^{\prime}\right)^{\frac{1}{n-k-1}} \leq & a\left(V^{\prime}\right)^{1-\delta_{1}}+c b t^{\frac{k-1}{k}} V^{\delta_{2}+\delta_{3}}\left(V^{\prime}\right)^{1-\delta_{2}}+\frac{c b}{1+\delta_{3}} t^{\frac{n-k-2}{n-k-1}}\left(V^{1+\delta_{3}}\right)^{\prime} \\
& +c|H| t^{\frac{k-1}{k}} V^{\frac{1}{k}}\left(V^{\prime}\right)^{1-\delta_{2}}+\alpha c|H| t^{\frac{n-k-2}{n-k-1}}\left(V^{1 / \alpha}\right)^{\prime}
\end{aligned}
$$


We now integrate both sides from 0 to $t$ as follows. Noting that $0 \leq \delta_{1}, \delta_{2}, \delta_{3}<1$ and using Hölder's inequality we get the inequalities

$$
\int_{0}^{t}\left(V^{\prime}\right)^{1-\delta_{1}} d s \leq t^{\delta_{1}}\left(\int_{0}^{t} V^{\prime} d s\right)^{1-\delta_{1}}=t^{\delta_{1}} V(t)^{1-\delta_{1}}
$$

and

$$
\begin{aligned}
\int_{0}^{t} s^{\frac{k-1}{k}} V^{\delta_{2}+\delta_{3}}\left(V^{\prime}\right)^{1-\delta_{2}} d s & \leq t^{\frac{k-1}{k}} \int_{0}^{t} V^{\delta_{2}+\delta_{3}}\left(V^{\prime}\right)^{1-\delta_{2}} d s \\
& \leq t^{\frac{k-1}{k}} t^{\delta_{2}}\left(\int_{0}^{t} V^{\left(\delta_{2}+\delta_{3}\right) /\left(1-\delta_{2}\right)}\left(V^{\prime}\right) d s\right)^{1-\delta_{2}} \\
& =t^{\frac{n-k-2}{n-k-1}}\left(\frac{1-\delta_{2}}{1+\delta_{3}}\right)^{1-\delta_{2}} V^{1+\delta_{3}} .
\end{aligned}
$$

Handling the integral of the fourth term on the right hand side of equation (5.8) in the same manner, we integrate equation (5.8) from 0 to $t$ to obtain

$$
\begin{aligned}
\left(V^{\prime}\right)^{1 / \alpha} \leq & \frac{1}{\alpha}\left(a t^{\delta_{1}} V^{1-\delta_{1}}+c b\left[\left(\frac{1-\delta_{2}}{1+\delta_{3}}\right)^{1-\delta_{2}}+\frac{1}{1+\delta_{3}}\right] t^{\frac{n-k-2}{n-k-1}} V^{1+\delta_{3}}\right) \\
& +\frac{c|H|}{\alpha}\left(\left[\left(1-\frac{\alpha}{k}\right)^{1-\delta_{2}}+\alpha\right] t^{\frac{n-k-2}{n-k-1}} V^{1 / \alpha}\right) .
\end{aligned}
$$

Noting that both quantities in brackets are bounded above by 2 and since $0<\alpha<1$ the inequality $(x+y)^{\alpha} \leq x^{\alpha}+y^{\alpha}$ for $x, y \geq 0$ implies

$$
V^{\prime} \leq \alpha^{-\alpha}\left(a t^{\delta_{1}} V^{1-\delta_{1}}+2 c b t^{\frac{n-k-2}{n-k-1}} V^{1+\delta_{3}}\right)^{\alpha}+(2 c|H| / \alpha)^{\alpha} t^{2 \alpha-1} V
$$

Multiplying by the integrating factor $\mu(t)=e^{-\kappa t^{2 \alpha}}$ where $\kappa=(2 c|H| / \alpha)^{\alpha} /(2 \alpha)$ transforms this inequality into

$$
(\mu V)^{\prime} \leq \alpha^{-\alpha}\left(a t^{\delta_{1}} V^{1-\delta_{1}}+2 c b t^{\frac{n-k-2}{n-k-1}} V^{1+\delta_{3}}\right)^{\alpha} \mu
$$

and using the fact that $0<\mu \leq 1$ for $t \geq 0$ we can write

$$
(\mu V)^{\prime} \leq \alpha^{-\alpha}\left(a t^{\delta_{1}}(\mu V)^{1-\delta_{1}}+2 c b t^{\frac{n-k-2}{n-k-1}}(\mu V)^{1+\delta_{3}}\right)^{\alpha} .
$$

Put $r_{0}=\inf \{r: b \mu(r) V(r) \geq 1\}$ with $r_{0}=\infty$ if $b \mu(r) V(r)<1$ for all $r>0$. Define the function $f:[0, \infty) \rightarrow \mathbb{R}$ by

$$
f(t)= \begin{cases}\mu(t) V(t) & \text { if } t \leq r_{0} \\ \max \{\mu(t) V(t), 1 / b\} & \text { if } t>r_{0}\end{cases}
$$

and observe that $f$ is absolutely continuous and satisfies the differential inequality (5.9) with $f$ in place of $\mu V$. We now use this inequality to derive an upper bound for the function $f(t)$. To integrate this inequality, first notice that the exponents satisfy $1-\delta_{2}<1+\delta_{3}$. On the interval $\left[0, r_{0}\right]$, since $b f \leq 1$ we thus have

$$
f^{\prime} \leq \alpha^{-\alpha}\left(a t^{\delta_{1}}+2 c b^{1-\delta_{1}-\delta_{3}} t^{\frac{n-k-2}{n-k-1}}\right)^{\alpha} f^{1-\frac{\alpha}{n-m-1}}, \quad\left(t \leq r_{0}\right) .
$$

Putting $\beta=\delta_{1}+\delta_{3}$ it follows that for $0 \leq t \leq r_{0}$ there holds

$$
\left(f^{\frac{\alpha}{n-m-1}}\right)^{\prime} \leq \frac{1}{n-m-1} \alpha^{1-\alpha}\left(a t^{\delta_{1}}+2 c b^{1-\beta} t^{\frac{n-k-2}{n-k-1}}\right)^{\alpha}, \quad\left(t \leq r_{0}\right) .
$$


Integrating the right hand side from 0 to $r \leq r_{0}$ using Hölder's inequality we find

$$
\int_{0}^{r}\left(a t^{\delta_{1}}+2 c b^{1-\beta} t^{\frac{n-k-2}{n-k-1}}\right)^{\alpha} d t \leq r^{1-\alpha}\left(\int_{0}^{r} a t^{\delta_{1}}+2 c b^{1-\beta} t^{\frac{n-k-2}{n-k-1}} d t\right)^{\alpha}
$$

and so carrying out the integration yields

$$
f(r) \leq\left(\frac{\alpha^{1-\alpha}}{n-m-1}\right)^{\frac{n-m-1}{\alpha}}\left(\frac{a}{1+\delta_{1}} r^{\frac{n-m}{n-m-1}}+\frac{2 c b^{1-\beta}}{2-\frac{1}{n-k-1}} r^{2}\right)^{n-m-1}, \quad\left(r \leq r_{0}\right) .
$$

Simplifying the expression, noting that $1 /\left(1+\delta_{1}\right) \leq 1,2-1 /(n-k-1) \geq 1$, and $\alpha^{1-\alpha} /(n-m-1) \leq 1$ we finally obtain

$$
f(r) \leq w(r)^{n-m-1}, \quad\left(r \leq r_{0}\right)
$$

where

$$
w(r)=\left(\frac{\alpha}{n-m-1}\right)^{\frac{1}{n-k-1}} \operatorname{vol}(\hat{\nu})^{\frac{1}{n-m-1}} r^{\frac{n-m}{n-m-1}}+2 c b^{1-\beta} r^{2} .
$$

For $t \geq r_{0}$ we may assume $b \neq 0$ and since $b f \geq 1$ we have

$$
f^{\prime} \leq \alpha^{-\alpha}\left(a b^{\delta_{1}+\delta_{3}} t^{\delta_{1}}+2 c b t^{\frac{n-k-2}{n-k-1}}\right)^{\alpha} f^{1-\frac{\alpha}{p}}, \quad\left(t \geq r_{0}\right) .
$$

Proceeding as above except that we integrate from $r_{0}$ to $r>r_{0}$ it is easy to check that for $r \geq r_{0}$,

$$
f(r) \leq \frac{1}{b}\left[1+b^{\frac{\alpha}{n-m-1}} w(r)^{\alpha}\right]^{p / \alpha} .
$$

Moreover, for $r \geq r_{0}$ we have $b w(r)^{n-m-1} \geq b w\left(r_{0}\right)^{n-m-1} \geq b f\left(r_{0}\right)=1$ and hence

$$
b^{-1}\left(1+b^{\frac{\alpha}{n-m-1}} w(r)^{\alpha}\right)^{p / \alpha} \leq b^{-1}\left(2 b^{\frac{\alpha}{n-m-1}} w(r)^{\alpha}\right)^{p / \alpha}=2^{p / \alpha} b^{\beta p} w(r)^{p}
$$

and thus for $r \geq r_{0}$ we have

$$
f(r) \leq 2^{p / \alpha} b^{\beta p} w(r)^{p}, \quad\left(r \geq r_{0}\right) .
$$

Combining equations (5.10) and (5.11) it then follows that for all $r \geq 0$ the function $f$ satisfies

$$
f(r) \leq w(r)^{n-m-1}+2^{p / \alpha} b^{\beta p} w(r)^{p} .
$$

Noting that $f(r) \geq \mu(r) V(r)$ it follows that the volume of the tube $T(\Sigma, r)$ satisfies

$$
V(r) \leq\left(w(r)^{n-m-1}+2^{p / \alpha} b^{\beta p} w(r)^{p}\right) e^{\kappa r^{2 \alpha}}
$$

This completes the proof of Theorem 1.1 contingent on our proof of Lemma 5.2.

Remark 5.3. As mentioned in the introduction, in the case of a pointwise lower curvature bound $\left\|\left(\rho_{k}\right)_{-}\right\|_{p}=0$ with $k=m$, the estimate reduces to

$$
V(r) \leq \frac{1}{n-m} \operatorname{vol}(\Sigma) \operatorname{vol}\left(\mathbb{S}^{n-m-1}\right) r^{n-m}
$$

which is precisely the volume of a tube around a piece of an $m$-plane in $\mathbb{R}^{n}$. The loss of sharpness in the pointwise case when $k \neq m$ comes from the use of Hölder's inequality above, and could be removed by setting $\left\|\left(\rho_{k}\right)_{-}\right\|_{p}=0$ earlier in the computation. 
Proof of Lemma 5.2. Define

$$
\begin{aligned}
& \sigma=\min \left\{\varphi_{+} / m, \psi_{+} /(n-m-1)\right\} \\
& \tau=\max \left\{\varphi_{+} / m, \psi_{+} /(n-m-1)\right\}
\end{aligned}
$$

and observe that both $\sigma$ and $\tau$ are absolutely continuous and from equation (3.2), using the fact that $0 \leq\left(\rho_{n-k-1}\right)_{-} \leq\left(\rho_{k}\right)_{-}$they satisfy

$$
\begin{aligned}
\sigma^{\prime}+\sigma^{2} & \leq\left(\rho_{k}\right)_{-} \\
\tau^{\prime}+\tau^{2} & \leq\left(\rho_{k}\right)_{-} .
\end{aligned}
$$

Multiplying the first equation by $(\sigma \tau)^{p-1} \mathcal{A}$ and integrating, we have

$$
\int_{0}^{r} \sigma^{\prime}(\sigma \tau)^{p-1} \mathcal{A} d t+\int_{0}^{r} \sigma^{p+1} \tau^{p-1} \mathcal{A} \leq \int_{0}^{r}\left(\rho_{k}\right)_{-}(\sigma \tau)^{p-1} \mathcal{A} d t .
$$

Integrating the first term by parts we find that

$$
\begin{gathered}
\frac{1}{p} \int_{0}^{r}\left(\sigma^{p}\right)^{\prime} \tau^{p-1} \mathcal{A} d t=\left.\frac{1}{p} \sigma^{p} \tau^{p-1} \mathcal{A}\right|_{0} ^{r}-\frac{p-1}{p} \int_{0}^{r} \sigma^{p} \tau^{p-2} \tau^{\prime} \mathcal{A} d t-\frac{1}{p} \int_{0}^{r} \sigma^{p} \tau^{p-1} h \mathcal{A} d t \\
\geq 0-\frac{p-1}{p} \int_{0}^{r} \sigma^{p} \tau^{p-2}\left(\left(\rho_{k}\right)_{-}-\tau^{2}\right) \mathcal{A} d t-\frac{n-k-1}{p} \int_{0}^{r} \sigma^{p} \tau^{p-1}(\sigma+\tau) \mathcal{A} d t
\end{gathered}
$$

where we have used the fact that $\sigma \tau$ is bounded as $t \rightarrow 0$ and $\mathcal{A}(0)=0$ for $m<n-1$. The last term uses the observation $h=\varphi+\psi \leq(n-k-1)(\sigma+\tau)$. Substituting back into (5.13), we now have

$$
\begin{aligned}
\frac{p-(n-k)}{p} \int_{0}^{r}(\sigma \tau)^{p} \mathcal{A} d t & +\left(1-\frac{n-k-1}{p}\right) \int_{0}^{r} \sigma^{p+1} \tau^{p-1} \mathcal{A} d t \\
& \leq \frac{p-1}{p} \int_{0}^{r}\left(\rho_{k}\right)_{-} \sigma^{p} \tau^{p-2} \mathcal{A} d t+\int_{0}^{r}\left(\rho_{k}\right)_{-}(\sigma \tau)^{p-1} \mathcal{A} d t
\end{aligned}
$$

Assuming $p>n-k$ the first term is positive, the second term is non-negative and can be dropped, and since $0 \leq \sigma \leq \tau$ we can use $\sigma^{p} \tau^{p-2} \leq(\sigma \tau)^{p-1}$ to obtain

$$
\int_{0}^{r}(\sigma \tau)^{p} \mathcal{A} d t \leq \frac{2 p-1}{p-(n-k)} \int_{0}^{r}\left(\rho_{k}\right)_{-}(\sigma \tau)^{p-1} \mathcal{A} d t .
$$

Finally, using Hölder's inequality on the right hand side we have

$$
\int_{0}^{r}\left(\rho_{k}\right)_{-}(\sigma \tau)^{p-1} \mathcal{A} d t \leq\left(\int_{0}^{r}\left(\rho_{k}\right)_{-}^{p} \mathcal{A} d t\right)^{1 / p}\left(\int_{0}^{r}(\sigma \tau)^{p} \mathcal{A} d t\right)^{1-\frac{1}{p}}
$$

and the lemma follows immediately.

We conclude with a proof of Corollary 1.4.

Proof of Corollary 1.4. Fix $n, m, p, H, v_{0}, D$ as in the statement of the Corollary. By Theorem 1.1, there exists a function $F(a, b, r)$ with the property that $F \rightarrow 0$ as $a, b \rightarrow 0$ such that for any closed $m$-dimensional minimal submanifold $\Sigma$ of a complete $n$-dimensional Riemannian manifold $M$ the volume of the tube around $\Sigma$ satisfies $\operatorname{vol}(T(\Sigma, r)) \leq F\left(\operatorname{vol}(\Sigma),\left\|\left(\rho_{k}-H\right)_{-}\right\|_{p}, r\right)$.

Given a closed minimal submanifold $\Sigma^{m}$ of an $n$-dimensional closed Riemannian manifold satisfying $\operatorname{vol}(M) \geq v_{0}$ and $\operatorname{diam}(M) \leq D$, since $M \subset T(\Sigma, D)$ we have

$$
v_{0} \leq \operatorname{vol}(M)=\operatorname{vol}(T(\Sigma, D)) \leq F\left(\operatorname{vol}(\Sigma),\left\|\left(\rho_{k}-H\right)_{-}\right\|_{p}, D\right) .
$$


Since $v_{0}$ is fixed, for sufficiently small $\epsilon$ there exists a number $\delta>0$ such that if $\left\|\left(\rho_{k}-H\right)_{-}\right\|_{p} \leq \epsilon$ then $\operatorname{vol}(\Sigma) \geq \delta$.

\section{REFERENCES}

1. Erwann Aubry, Finiteness of $\pi_{1}$ and geometric inequalities in almost positive Ricci curvature, Ann. Sci. École Norm. Sup. (4) 40 (2007), no. 4, 675-695. MR 2191529

2. Richard L. Bishop and Richard J. Crittenden, Geometry of manifolds, Pure and Applied Mathematics, Vol. XV, Academic Press, New York-London, 1964. MR 0169148

3. Simon Brendle, Constant mean curvature surfaces in warped product manifolds, Publications mathématiques de l'IHÉS 117 (2013), no. 1, 247-269.

4. Sylvestre Gallot, Isoperimetric inequalities based on integral norms of Ricci curvature, Astérisque (1988), no. 157-158, 191-216, Colloque Paul Lévy sur les Processus Stochastiques (Palaiseau, 1987). MR 976219

5. Alfred Gray, Tubes, second ed., Progress in Mathematics, vol. 221, Birkhäuser Verlag, Basel, 2004, With a preface by Vicente Miquel. MR 2024928

6. X. Gual-Arnau and R. Masó, Mean curvature comparison for tubular hypersurfaces in symmetric spaces, Balkan J. Geom. Appl. 8 (2003), no. 1, 53-62, Volume dedicated to the memory of Grigorios Tsagas (1935-2003), president of the Balkan Society of Geometers (1997-2003). MR 2030321

7. L. Guijarro and F. Wilhelm, Focal radius, rigidity, and lower curvature bounds, Proceedings of the London Mathematical Society 116 (2018), no. 6, 1519-1552.

8. Ernst Heintze and Hermann Karcher, A general comparison theorem with applications to volume estimates for submanifolds, Ann. Sci. École Norm. Sup. (4) 11 (1978), no. 4, 451-470. MR 533065

9. Christian Ketterer and Andrea Mondino, Sectional and intermediate Ricci curvature lower bounds via optimal transport, Adv. Math. 329 (2018), 781-818. MR 3783428

10. Peter Li and Jiaping Wang, Comparison theorem for Kähler manifolds and positivity of spectrum, J. Differential Geom. 69 (2005), no. 1, 43-74. MR 2169582

11. Carlo Mantegazza and Andrea Carlo Mennucci, Hamilton-Jacobi equations and distance functions on Riemannian manifolds, Appl. Math. Optim. 47 (2003), no. 1, 1-25. MR 1941909

12. P. Petersen, S. D. Shteingold, and G. Wei, Comparison geometry with integral curvature bounds, Geom. Funct. Anal. 7 (1997), no. 6, 1011-1030. MR 1487752

13. P. Petersen and G. Wei, Relative volume comparison with integral curvature bounds, Geom. Funct. Anal. 7 (1997), no. 6, 1031-1045. MR 1487753

14. Peter Petersen, Riemannian geometry, third ed., Graduate Texts in Mathematics, vol. 171, Springer, Cham, 2016. MR 3469435

15. Peter Petersen and Chadwick Sprouse, Integral curvature bounds, distance estimates and applications, J. Differential Geom. 50 (1998), no. 2, 269-298. MR 1684981

16. Peter Petersen and Frederick Wilhelm, On Frankel's theorem, Canad. Math. Bull. 46 (2003), no. 1, 130-139. MR 1955620

17. Guohuan Qiu and Chao Xia, A generalization of Reilly's formula and its applications to a new Heintze-Karcher type inequality, Int. Math. Res. Not. IMRN (2015), no. 17, 7608-7619. MR 3403995

18. Antonio Ros, Compact hypersurfaces with constant higher order mean curvatures, Rev. Mat. Iberoamericana 3 (1987), no. 3-4, 447-453. MR 996826

19. Zhong Min Shen, On complete manifolds of nonnegative kth-Ricci curvature, Trans. Amer. Math. Soc. 338 (1993), no. 1, 289-310. MR 1112548

20. Zhong Min Shen and Guofang Wei, Volume growth and finite topological type, Differential geometry: Riemannian geometry (Los Angeles, CA, 1990), Proc. Sympos. Pure Math., vol. 54, Amer. Math. Soc., Providence, RI, 1993, pp. 539-549. MR 1216645

21. Frederick Wilhelm, On intermediate Ricci curvature and fundamental groups, Illinois J. Math. 41 (1997), no. 3, 488-494. MR 1458186

22. Guoqiang $\mathrm{Wu}$ and Rugang Ye, A note on Obata's rigidity theorem, Commun. Math. Stat. 2 (2014), no. 3-4, 231-252. MR 3326231

23. H. Wu, Manifolds of partially positive curvature, Indiana University Mathematics Journal 36 (1987), no. 3, 525-548. 
24. Hong-Wei Xu and Fei Ye, Differentiable sphere theorems for submanifolds of positive $k$-th Ricci curvature, Manuscripta Math. 138 (2012), no. 3-4, 529-543. MR 2916324

25. Deane Yang, Convergence of Riemannian manifolds with integral bounds on curvature. I, Ann. Sci. École Norm. Sup. (4) 25 (1992), no. 1, 77-105. MR 1152614

University of California, Santa Barbara

E-mail address: ychahine@math.ucsb.edu 